\title{
Vecchie motivazioni e nuove proposte per l'emodialisi domiciliare
}

\author{
Moreno Malaguti, Marco Biagini
}

\author{
Dipartimento di Nefro-Urologia, ASL RM F, Ospedale San Paolo, Civitavecchia
}

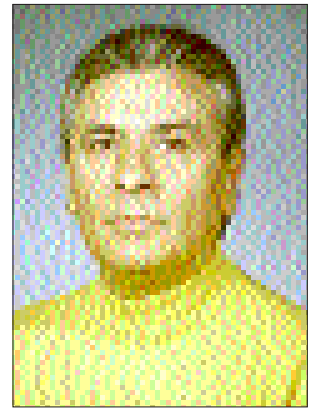

Moreno Malaguti
$\mathbf{T}$ utti sappiamo che il numero degli emodializzati è in continuo aumento per l'invecchiamento della popolazione, del cambiamento delle patologie che portano all'insufficienza renale, della maggiore sopravvivenza dei pazienti e dell'esiguità numerica dei trapianti eseguiti (1). È inoltre difficile espandere i Centri ospedalieri o crearne dei nuovi a causa del peso economico che rappresentano per le risorse dedicate alla Sanità; eppure molti pazienti non hanno le caratteristiche di chi chiede passivamente assistenza, ma desiderano un accesso ospedaliero limitato per sentirsi riabilitati e migliorare la qualità della vita (2-5). Quest'aspettativa può essere realizzata anche sviluppando l'emodialisi domiciliare (EDD), ma nonostante il favore incontrato nella letteratura scientifica (per quantificarlo basta una ricerca bibliografica su Internet) sull'efficacia della tecnica (6-8) e sulla riduzione dei costi (9), nessuno passa alla fase organizzativa. Gli Assessorati re- gionali alla Salute pubblica, pur obbligando i Centri di Riferimento a indirizzare una percentuale di pazienti al trattamento domiciliare sia peritoneale (DP) sia emodialitico (HD) (10), non prevedono fondi per l'acquisto delle attrezzature per la EDD, disincentivando economicamente $\mathrm{i}$ pazienti.

Esistono poi motivazioni contrarie alla EDD legate all'età media elevata dei pazienti, meno autonomi in manualità indispensabili, con patologie comorbide che determinano complicanze intradialitiche quali ipotensioni, aritmie e crampi e con minore motivazione verso l'autogestione del proprio tempo perché non impegnati in attività lavorative. Infine il tessuto sociale e familiare, sempre meno compatto intorno all'anziano, rappresenta un ostacolo a trovare dei partner che collaborino attivamente nell'esecuzione del trattamento.

I Paesi con ampi territori scarsamente popolati sviluppano piani per la " $h o$ me-based care" delle malattie croniche (11), affidando la cura dei pazienti che non necessitano d'assistenza intensiva alla famiglia o a strutture sanitarie decentrate, dove una singola figura professionale, debitamente addestrata, gestisce il trattamento di molti malati con ottimi risultati sia clinici (12) sia economici (13).

I cambiamenti sociali suesposti impongono una riorganizzazione dell'emodialisi, con la creazione di una maggiore flessibilità assistenziale che la porti più vicina all'utente per migliorarne la qualità limitando il costo. E necessario ribaltare la situazione: da un sistema che convoglia tutti i nefropatici in grandi Centri, dove tanto personale, esperto e competente, li dializza in modo "industriale", con tempi prefissati, chiedendogli accettazione passiva del trattamento senza il rispetto dovuto alle loro necessità e sensibilità, a un sistema dove un limitato staff dedicato crea i presupposti per una terapia consapevole e gestita in accordo, nei luoghi e tempi più consoni alle esigenze del singolo paziente.

I Centri che fanno DP conoscono questa organizzazione, ma nessuno l'ha mai applicato alla EDD, anche perché è idea comune che HD e DP abbiano differenti necessità. Forse questo è vero sul piano operativo, ma la parte organizzativa può essere comune e funzionare a dovere.

Bisogna istituire gli ambulatori di pre-dialisi, che hanno il compito di offrire e indirizzare il paziente al trattamento a lui più idoneo. Una volta che la EDD è stata scelta, il paziente 
deve essere seguito da personale slegato dall'emodialisi ospedaliera ma dipendente dal Centro, dedicato e fortemente motivato, per effettuare l'addestramento e l'istruzione sua e dell'eventuale partner. Fra i compiti di questo personale deve esserci quello della mobilità sul territorio per assistere i pazienti che non hanno la possibilità di avere un partner attivo collaborante o ricoverati presso RSA, lungo-degenze e case di riposo, non per l'intera esecuzione del trattamento, ma per le manualità che il paziente non può espletare autonomamente. Bisogna quindi creare anche una nuova figura d'operatore sanitario, che abbia, oltre alle conoscenze professionali infermieristiche e tecniche dell'emodialisi, anche la cultura dell'assistenza sul territorio, che conosca le tecniche psicologiche per interagire con pazienti spesso fragili, per diventare un riferimento, una figura empatica che sappia infondere sicurezza e fiducia a chi deve affrontare, spesso da solo, un trattamento altamente tecnologico quale una depurazione extracorporea, che sappia rispondere ai dubbi e indirizzare alle scelte migliori i pazienti seguiti. Questa figura, condivisa da più pazienti, crea i presupposti per una migliore qualità globale permettendo, contemporaneamente, una riduzione dei costi dovuti al personale che, come tutti sanno, rappresenta il capitolo di spesa più consistente.

Trovo giusto prevedere per questi operatori un inquadramento maggiormente incentivante sul piano economico sia in sede contrattuale nazionale che decentrata presso l'Azienda d'appartenenza, al limite con ripartizione parziale delle somme risparmiate tramite la loro opera.

Dietro a questi operatori deve esserci un supporto logistico adeguato. Il Centro di Riferimento deve essere dotato di sistemi telematici con cui controllare l'andamento del trattamento su richiesta dei pazienti (14) e di linee telefoniche dedicate, per colloquiare con loro, valutare le difficoltà incontrate e aiutarli a risolvere autonomamente i problemi.

Anche la figura del medico cambia, è sufficiente un'unità per controllare un alto numero di pazienti che sono valutati, programmaticamente, presso il Centro con cadenza variabile secondo le condizioni cliniche dei pazienti.

È poi indispensabile pensare alle attrezzature. L'industria deve realizzare monitor di semplice utilizzo e gestione, che non richiedano complicati procedimenti di pulizia-disinfezione e che abbrevino e rendano semplice, per quanto possibile, la loro preparazione. Inoltre non tutti possono permettersi di acquistare in proprio un monitor di preparazione e un sistema di trattamento dell'acqua con osmosi inversa; se non esistono e non sono reperibili fondi per sollevare i pazienti da queste spese, è necessario coinvolgere le industrie del settore spingendole a fornire, al limite, dei "service" che includano le attrezzature necessarie, la loro manutenzione, i materiali e relativa consegna al domicilio e lo smaltimento controllato dei rifiuti speciali. Non bisogna, infine, dimenticare che EDD significa anche un magazzino da gestire con una quantità e tipologia di materiali non indifferente, che richiede organizzazione, spazi e tempi. Ma su quest'argomento non ho dubbi che un accordo si troverebbe.

I vantaggi che si possono ottenere sono tanti: oltre a quelli economici, conosciuti da tutti, bisogna considerare che per il paziente anziano la necessità di concentrarsi in un'attività importante, quale la tutela della propria salute anche in termini di manualità, è fonte di maggiore autostima e di minore pericolo di sindromi depressive, tanto temibili. I sintomi intradialitici possono essere minimizzati allungando il tempo di dialisi, che al domicilio non presenta i problemi organizzativi del trattamento in Centro, modulando così l'ultrafiltrazione ed eliminando la maggior parte delle complicanze come dimostrato dall'esperienza di Tassin. Infine l'ambiente domestico è sicuramente più gradito $\mathrm{e}$ non obbliga ai viaggi di trasporto dal domicilio al Centro e viceversa, che rappresentano un elemento di disagio e un ulteriore aggravio di spesa.

Il nostro Centro, probabilmente per motivi geografici e sociali, si è trovato nella strana posizione di ricevere un numero crescente di richieste d'appoggio tecnico-clinico-amministrativo a pazienti che volevano effettuare EDD, utenti che prima afferivano a strutture della Capitale e che non riuscivano a convivere decentemente con la loro terapia cronica. Questo perché gli orari obbligati non permettono una vita lavorativa piena, o perché il contatto con gli altri pazienti è fonte di disagio psicologico o perché, molto più semplicemente, abitando più di un domicilio, non avevano assistenza continua ovunque essi fossero. Nella nostra esperienza di sei unità in EDD, pari al $7.5 \%$ dei pazienti del nostro Dipartimento, solo due sono giovani con una buona condizione di salute, gli altri sono anziani (età media $62 \pm 14$ anni contro i $65 \pm 13$ della popolazione in Centro), con patologie extrarenali quali cardiopatie ischemiche o degenerative, arteriopatie obliteranti periferiche, ma con una sola grande motivazione comune: sentirsi liberi.

Di questi pazienti uno in particolare rappresenta appieno lo spirito della EDD.

C.G., paziente di 51 anni, in dialisi da 26 a causa di GNF cronica. Dopo i primi cinque anni di dialisi presso un Centro convenzionato, passa alla EDD nel 1980. In questo periodo il paziente ha seguito un regime dialitico "originale" sottoponendosi al trattamento ogni tre giorni per sei ore: non necessita di EPO, supplementi marziali, antiipertensivi o chelanti del fosforo. A conti fatti, questo signore fa 60 ore mensili di HD con 10 trattamenti rispetto alle 52 ore del ritmo trisettimanale con 13 trattamenti. Le otto ore di differenza significano due sedute emodialitiche in più. Il tempo speso in dialisi è recuperato con il tempo risparmiato nella preparazione del monitor e dei materiali visto il minor numero di trattamenti.

malamore@tin.it

nefrocv@tin.it 


\section{BIBLIOGRAFIA}

1. Comitato Nazionale per il Registro di Dialisi e Trapianto: Report del Registro, anno 1998. RIDT 98 , Taormina 2000 .

2. Cameron JI, Whiteside C, Katz J, Devins GM. Differences in quality of life across renal replacement therapies: a meta-analy tic comparison. Am J Kidney Dis 2000; 35: 629-37.

3. Morris PL, Jones B. Life satisfaction across treatment methods for patients with end-stage renal failure. Med J Aust 1989; 150: 428-32.

4. Oberley ET, Schatell DR. Home hemodialysis: survival, quality of life, and rehabilitation. Adv Ren Replace Ther 1996; 3: 147-53.

5. Korevaar JC, Jansen MA, Merkus MP, Dekker FW, Boeschoten EW, Krediet RT. Quality of life in predialysis end-stage renal disease patients at the initiation of dialysis therapy. The NECOSAD Study Group. Perit Dial Int 2000; 20: 69-75.

6. Mailloux LU, Kapikian N, Napolitano B, et al. Home hemodialysis: patient outcomes during a 24 year period of time from 1970 through 1993. Adv Ren Replace Ther 1996; 3: 112-9.

7. Grant AC, Rodger RS, Howie CA, Jun or BJ, Briggs JD, Macdougall AI. Dialysis at home in the west of Scotland: a comparison of hemodialysis and continuous ambulatory peritoneal dialysis in age- and sex-matched controls. Perit Dial Int 1992; 12: 365-8.

8. Woods JD, Port FK, Stannard D, Blagg CR, Held PJ. Comparison of mortality with home hemodialysis and center hemodialysis: a national study. Kidney Int 1996; 49: 146470.

9. De Vecchi AF, Dratwa M, Wiedermann ME. Health care systems and end-stage renal disease (ESRD) the- rapies. An International review: costs and reimbursement/funding of EESRD therapies. Nephrol Dial Transplant 1999; 6 (suppl): S31-

10. ${ }^{41}$ Piano Sanitario delle Regione Lazio, D.G.R. n. 619 del 03/03/1998.

11. Wellard SJ, Street AF. Family issues in home-based care. Int J Nurs Pract 1999; 5: 132-6.

12. Arkouche W, Traeger J, Delawari E, et al. Twenty-five years of experience with out-center hemodialysis. Kidney Int 1999; 56: 226975 .

13. Goeree R, Manalich J, Grootendorst P, Beecroft ML, Churchill DN. Cost analysis of dialysis treatments for end-stage renal disease (ESRD). Clin Invest Med 1995; 18: 455-64.

14. Agroyannis B, Fourtounas C, Romagnoli G, et al. Telemedicine technology and applications for home hemodialysis. Int J Artif Organs 1999; 22: 679-83. 